\title{
Mediation: incomplete information bargaining with filtered communication
}

\author{
Xavier Jarque $^{\mathrm{a}, *}$, Clara Ponsatí ${ }^{\mathrm{b}}$, József Sákovics $^{\mathrm{c}}$ \\ a Departament d'Economia i d'Història Econòmica, E.U.E.E, Universitat Autònoma de Barcelona, \\ 08193 Bellaterra, Barcelona, Spain \\ b Institut d'Anàlisi Econòmica, CSIC and CODE, Universitat Autònoma de Barcelona, \\ 08193 Bellaterra, Barcelona, Spain \\ ${ }^{\mathrm{c}}$ Edinburgh School of Economics, University of Edinburgh, 50 George Square, Edinburgh EH8 9JY, UK
}

Received 18 January 2002; received in revised form 27 March 2003; accepted 29 March 2003

\begin{abstract}
We analyze a continuous-time bilateral double auction in the presence of two-sided incomplete information and a smallest money unit. A distinguishing feature of our model is that intermediate concessions are not observable by the adversary: they are only communicated to a passive auctioneer. An alternative interpretation is that of mediated bargaining. Perfect Bayesian Equilibria are characterized. We show that an equilibrium using only the extreme agreements always exists and display the necessary and sufficient condition for the existence of equilibra which yield intermediate agreements. For the symmetric case with uniform-type distribution we numerically calculate the equilibria. We find that the equilibrium which does not use compromise agreements is the least efficient, however the social welfare of the rest of the equilibria is decreasing in the number of compromise agreements used.
\end{abstract}

(C) 2003 Elsevier Science B.V. All rights reserved.

Keywords: Mediation; Continuous time; Bargaining

\section{Introduction}

In this paper, we present an in-depth analysis of mediation in bargaining. Mediation being a wide-spread practice - from international negotiations to divorce proceedings - this is empirically relevant in its own right. Our concurrent intention is more theoretical though: to provide an alternative approach to the study of bargaining with incomplete information.

\footnotetext{
* Corresponding author. Tel.: +34-935-8118-02; fax: +34-935-8120-12.

E-mail addresses: xavier.jarque@uab.es (X. Jarque), clara.ponsati@uab.es (C.Ponsatí),jozsef.sakovics@ed.ac.uk (J. Sákovics).
} 
The difficulties encountered by this literature are mostly due to the issues arising from unrestricted—or, arbitrarily restricted—conditional belief structures, which, unfortunately, arise most naturally in dynamic games of incomplete information. Instead of constraining the ways in which beliefs can be updated, we work with an extensive form that exogenously restricts the generation of the information events, which would trigger the formation of new posteriors. In particular, we assume that all offers that do not lead to agreement are unobservable. The easiest way to think of this situation is to say that it is a dynamic double auction: ${ }^{1}$ the players keep sending offers to an auctioneer, who only reveals them once they are compatible, and therefore an agreement has been reached. Within this context, we are able to give a complete characterization, as well as a number of interesting insights.

Following Zeuthen (1930), we consider the bargaining problem as a process of mutual concessions. While this seems to be the most natural interpretation, it is seldom an equilibrium feature in the literature. As in Bishop (1964)—within the context of a concession game- the force driving our negotiators to concede is impatience. The major difference is that we do not make any ad hoc assumption about how impatience is related to the rate of concessions. Instead, we present a full-fledged game-theoretic model, where the speed of conceding arises as an equilibrium phenomenon. Since we also generalize to incomplete information, the Hicks (1932) argument, that the negotiators can calculate what would be the eventual agreement and implement it without delay, does not apply in our context. In our model, delay is real, since it serves as a screening/signaling device (c.f. Admati and Perry, 1987).

Our paper can also be read as a study focused on the analysis of mediated bargaining. Mediation is generally seen as a useful tool to improve on the efficiency of negotiations. On one hand, it may make agreement viable in situations where otherwise there would be an impasse. On the other hand, it may lead negotiators to speed up mutual concessions, thereby decreasing the inefficient delay in reaching an agreement. As John T. Dunlop, former US Secretary of Labor and one of the foremost authorities on dispute resolution, explains:

The mediator has a special opportunity in the "end-game" of negotiations .... The critical problem is ... that any move may create the impression of being willing to move all the way to the position of the other side .... In these circumstances a third party may greatly facilitate agreement. The separate conditional acceptance to the mediator by one side of a proposal does not prejudice the position of that side if there is no agreement. It is not unusual for a mediator to secure the separate acceptance of each side ... and then to bring the parties together to announce that, even if they do not know it, they have an agreement.

(Dunlop, 1984, pg. 24)

We carry out our analysis in the framework of a model with four identifying characteristics: (i) two-sided incomplete information (about reservation values); (ii) two-sided offers; (iii) a discrete set of feasible agreements; and (iv) continuous time.

\footnotetext{
${ }^{1}$ See Chatterjee and Samuelson (1983), Broman (1989), Leininger et al. (1989), Satterthwaite and Williams (1989) and Gresik (1996) — among others—-for static models of bargaining, using the bilateral double auction mechanism.
} 
The immediate efficient agreement predicted by most models under complete information seems to be at odds with the perception that delays and impasses are common in all kinds of negotiations. Indeed, most real life bargaining situations are best described as games with two-sided private information. In addition, the essence of mediation is that the negotiators can take actions without their opponents knowing. However, in a complete information setup, in equilibrium, nothing could remain secret.

The simplest dynamic model of bargaining with two-sided private information is the one where only one of two extreme proposals can prevail: the war of attrition. Under mild assumptions, ${ }^{2}$ the war of attrition has a unique equilibrium. This is mostly due to the fact that it is a game of timing rather than one of bids. Interesting bargaining models, however, must contemplate the possibility of compromise agreements. In the standard models of bargaining with incomplete information where compromise agreements are feasible, a player offering a concession to her opponent incurs costs of two different types-she bears right away the direct cost of accepting a smaller portion of the surplus and, in addition, she weakens her bargaining position by having revealed herself as weaker than her opponent. These phenomena generate immense problems of belief updating that result in sets of multiple equilibria that are very difficult to characterize. Thus, in spite of the natural appeal of bargaining models with two-sided incomplete information, clear-cut general results have remained elusive. ${ }^{3}$

We overcome these difficulties by importing the advantages of the war of attrition approach: we examine a continuous-time bargaining game with a finite number of possible agreements. Apart from the fact that this is a more realistic scenario-the existence of a smallest money unit and the non-existence of a smallest time unit are facts-it is also technically advantageous. Once the finite set of agreements is fixed, one can again think of the bargaining game as one of timing: all the players have to decide is the speed at which they should make consecutive concessions. This point of view drastically simplifies the problem and it makes possible to characterize the equilibrium strategies as solutions to a system of ordinary differential equations. Additionally, this extensive form is rather robust, since it is practically "procedure free" (c.f. Ponsatí (1992, 1997), Perry and Reny (1993), Sákovics (1993) and Abreu and Gul (2000)).

We model the auctioneer/mediator in the simplest possible way. He is not an active player, his only role is to restrict the information flow between the negotiating parties (and to enforce that the agents honor their offers). At every date $t$, players confidentially report to the mediator the agreements they are willing to accept. The mediator remains passive while the players make insufficient concessions but announces the agreement as soon as it is reached. Since players do not observe the partial concessions of their opponent, the updating of beliefs is greatly simplified and we are able to attain a full characterization of the Perfect Bayesian Equilibrium (PBE) outcomes.

Players may decide to ignore the possibility of non-revealing partial concessions offered by the presence of the mediator, and thus a PBE that reproduces the outcome of the war of attrition, where only the two extreme proposals are feasible, always exists. We explore

\footnotetext{
2 See Ponsatí and Sákovics (1995).

3 See Fudenberg and Tirole (1983), Cramton (1984, 1992), Chatterjee and Samuelson (1987), Cho (1990), Ausubel and Deneckere (1992b, 1993), Ponsatí (1997) and Watson (1998) for some valuable partial results.
} 
the set of PBE further and show that the mediator does play a positive role in generating compromise outcomes: in addition to the war of attrition equilibrium (WAE), there also exists an ex post efficient ${ }^{4}$ Mediation Equilibrium (ME) — whenever the relative weight of the types who cannot make any concession is not too high. In the ME, agreements occur with positive probability at all the feasible prices and in all time intervals.

The above result is perhaps best interpreted in the light of the findings of Ponsatí (1997). She examines the face-to-face version of our bargaining game with three feasible agreements and shows that if players use Markov strategies (that is, they choose their action at each stage based only on current proposals and beliefs) then face-to-face bargaining yields the same outcome as a war of attrition in which only the extreme agreements are possible. ${ }^{5}$ That is, since in the current model the restriction to Markov strategies would play no role, we can conclude that if we expect the players to use such strategies, mediation can lead to an outcome where more agreements are used and thus it improves ex post efficiency. On the other hand, she also shows that on all the other equilibrium paths (involving compromise offers) there must exist intervals of time, where no concessions are made. Consequently, without the restriction to Markov strategies, mediation improves ex ante efficiency. ${ }^{6}$

The set of equilibria is completed by a unique ex post efficient (relative to the subset of agreements they employ) equilibrium for each subset of the feasible agreements containing the two extreme ones. When the distribution of types is very skewed, some ex post inefficient equilibria may also exist.

To obtain further insight, we also calculate (numerically) the equilibrium strategies and the resulting (utilitarian) social welfare for the case of symmetric and uniform-type distributions. Our most important findings are that the more agreements are used in equilibrium, the stricter are the necessary support conditions for existence and the lower is social welfare. That is, ex ante and ex post efficiency are antagonical.

Abreu and Gul (2000) also propose a "war-of-attrition-based" approach to the modelling of bargaining games without complete information. Replacing uncertainty about some fundamentals (reservation values, discount factors, etc.) they assume that the uncertainty is "strategic": with some positive probability, each player is of the irrational type, and will not change his initial demand. ${ }^{7}$ This assumption corresponds to the opposite extreme to ours: rather than filtering the information flow, they make information events so revealing, that once a player makes any concession, in equilibrium, she must fully concede.

The rest of the paper is organized as follows. Section 2 formally presents the model. In Section 3, we derive some preliminary results. The characterization of the set of equilibria is in Section 4. Section 5 exhibits the numerical solutions and the welfare analysis. Section 6 concludes. The proofs not presented in the text are relegated to Appendix A.

\footnotetext{
${ }^{4}$ Relative to the feasible prices, of course.

5 Note that this result is consistent with the finding of Ausubel and Deneckere (1992a) that in a game of one-sided incomplete information, the informed player prefers to remain silent: in the two-sided case both prefer not to make intermediate concessions.

6 Ausubel and Deneckere (1993) showed that, if the agents are allowed to use strategies that are non-monotonic in their valuation, the ex ante efficiency of the equilibrium can approximate the welfare bound imposed by incentive compatibility. It is questionable though, whether such strategies are indeed commonly used.

7 See Inderst (1999) for a model where both types of uncertainties are present.
} 


\section{The mediated bargaining game}

We study the following bargaining situation. Two players $(i=1,2)$ bargain about how to share some potential surplus that will be available only if and when they reach an agreement. We denote an agreement by a number, $x \in(0,1)$, that indicates the portion of the surplus assigned to Player 1, while Player 2 receives $1-x$. Feasible proposals and agreements are restricted to elements of the set $X=\left\{x^{1}, \ldots, x^{R}\right\}$ where $0<x^{1}<\cdots<x^{r}<\cdots<$ $x^{R}<1$.

An agreement can be reached at any time $t \in[0, \infty)$. Let $\left(x^{r}, t\right)$ denote an outcome of the bargaining, where $x^{r} \in X$ is the agreement and $t$ is the date at which it is reached. Define $s_{1}$ and $s_{2}$ as reservation values of Players 1 and 2, respectively. Then the outcome, $\left(x^{r}, t\right)$, gives a payoff of $u_{1}^{s_{1}}\left(x^{r}, t\right)=\left(x^{r}-s_{1}\right) \mathrm{e}^{-z_{1} t}$ to Player 1 of type $s_{1}$, while Player 2 of type $s_{2}$ receives $u_{2}^{s_{2}}\left(x^{r}, t\right)=\left(1-x^{r}-s_{2}\right) \mathrm{e}^{-z_{2} t}$, where the $z_{i}(>0)$ denote the players' discount rates. If offers never become compatible the outcome is perpetual disagreement, yielding payoff 0 to both players, regardless of their type. An easy way to think about this situation is to say that type $s_{1}$ of Player 1 is the seller of an item which she values at $s_{1}$, while type $s_{2}$ of Player 2 is the buyer who values the item at $1-s_{2}$ and they are bargaining over the price, $x^{r}$, the buyer should pay the seller in exchange for the item.

The reservation values are private information. They are the realizations of independent draws from the probability distributions $F_{i}\left(s_{i}\right), i=1,2$. These distribution functions are common knowledge and have positive densities $f_{i}$ on $\left[s_{i}^{L}, s_{i}^{H}\right], i=1,2$. We require that $s_{1}^{L}<x^{1} \leq 1-s_{2}^{H}<x^{2}$ and $x^{R-1}<s_{1}^{H} \leq x^{R}<1-s_{2}^{L}$. First it is not common knowledge that one of the players can surely offer an interior agreement. Second for each player there is at least one acceptable agreement (we rule out types that cannot get a positive surplus from any agreement, since they will never show up at the bargaining table anyway). Moreover, for each type there is a positive probability that gains from trade are possible. Finally, there are masses of types who cannot yield from their most preferred agreement and who can accept their least preferred agreement, respectively. ${ }^{8}$ A natural way in which this situation can arise is that the set of relevant feasible agreements are endogenously determined as a function of the distribution of types.

Our bargaining procedure has the following rules. The traders send private messages (sealed bids, if you will) to a mediator, whose only active role is to make the agreement public as soon as it is reached. The content of a message is the price at which the trader is willing to trade. As time goes by, the players can change their proposals at any point. We only impose the restriction that they have to honor any previously made offer. That is, we-or the mediator, if you will—do not allow offers to be withdrawn. Consequently, the demands of both players have to be non-increasing over time. ${ }^{9}$

\footnotetext{
8 The latter assumption rules out the trivial equilibrium, which consists of making non-serious offers (never to concede). At the same time it ensures the existence of a war-of-attrition-like equilibrium, see Corollary 1.

9 Technically speaking, this assumption causes a loss of generality. However, the resulting player behavior is much more plausible under this assumption than when it is not satisfied. With unrestricted strategies, the players would not hold compromise offers for any positive amount of time. Instead they would periodically "sample" whether their opponent is currently offering a compromise. Apart from the inefficiency and the huge multiplicity that this would generate, these equilibria would also require an important degree of coordination between the parties.
} 
A (pure) strategy for Player $i, \sigma_{i}$, is a measurable function from $\left[s_{i}^{L}, s_{i}^{H}\right] \times[0, \infty)$ to $X, i=1,2$, specifying for every type and every point in time the player's standing proposal. Note that concessions need not be made one by one. For instance, a strategy for Player 1 can "jump" at some time $t$ from proposal $x^{k}$ to proposal $x^{l}$ with $l<k-1$.

Since strategies are monotone in time and there are a finite number of feasible prices, the strategies are continuous in time except at a finite number of $t$ 's. At these points, we assume right-continuity (that is, $\left.\sigma_{i}(s, t)=\lim _{\mathcal{\varepsilon} \downarrow 0}\left(\sigma_{i}(s, t+\varepsilon)\right)\right)$ and thus we ensure the existence of a first time at which a proposal $x^{r} \in X$ is made. Consequently, a strategy can also be described using a vector-valued function identifying the first time the player makes each feasible proposal. To this effect, we let $\mu_{i}:\left[s_{i}^{L}, s_{i}^{H}\right] \rightarrow[0, \infty]^{R}, i=1,2$ satisfying

(a) $\mu_{1}^{R}\left(s_{1}\right)=0$ and $\mu_{1}^{k}\left(s_{1}\right) \leq \mu_{1}^{k-1}\left(s_{1}\right), k=2, \ldots, R$,

(b) $\mu_{2}^{1}\left(s_{2}\right)=0$ and $\mu_{2}^{k}\left(s_{2}\right) \leq \mu_{2}^{k+1}\left(s_{2}\right), k=1, \ldots, R-1$,

where the inequalities are strict if and only if proposal $x^{i}$ is held for a positive length of time. An equivalent representation for $\sigma_{1}$ is then as follows (a similar representation applies to $\sigma_{2}$ )

$$
\sigma_{1}(s, t)=\left\{\begin{array}{ccc}
x^{R}, & & \text { for } t<\mu_{1}^{R-1}(s), \\
x^{k}, & & \text { for } \quad \mu_{1}^{k}(s) \leq t<\mu_{1}^{k-1}(s), \\
& \ldots & \\
x^{1}, & & \text { for } t \geq \mu_{1}^{1}(s) .
\end{array}\right.
$$

Given a pair of types, $\left(s_{1}, s_{2}\right)$, a strategy profile determines a unique outcome of the game denoted by $\left(x\left(\sigma, s_{1}, s_{2}\right), t\left(\sigma, s_{1}, s_{2}\right)\right)$. To define this outcome, we adopt the convention that if, when the offers first become compatible, that is, Player 1 is proposing $x^{k}$ while Player 2 is proposing $x^{l}, k \leq l$, they are inefficient (i.e. $l>k$ ), then the resulting outcome is agreement at the offer that was made first, but naturally at the time when the later one was made. ${ }^{10}$

A system of beliefs for Player $i, \beta_{i}$, maps each point in time into some probability distribution on the support $\left[s_{j}^{L}, s_{j}^{H}\right]$ of types of Player $j, j \neq i$. Given a strategy-belief profile $(\sigma, \beta)$, let $u_{i}^{s_{i}}\left(\sigma, \beta_{i}, t\right)$ denote the expected payoff to Player $i$ of type $s_{i}$ conditional on having followed her strategy and not having arrived at an agreement by $t$. That is,

$$
u_{1}^{s_{1}}\left(\sigma, \beta_{1}, t\right)=\int_{\left[s_{2}^{L}, s_{2}^{H}\right]}\left(x\left(\sigma, s_{1}, v\right)-s_{1}\right) \mathrm{e}^{-z_{1} t\left(\sigma, s_{1}, v\right)} \mathrm{d} \beta_{1}(v ; t)
$$

and

$$
\left.u_{2}^{s_{2}}\left(\sigma, \beta_{2}, t\right)=\int_{\left[s_{1}^{L}, s_{1}^{H}\right]}\left(1-x\left(\sigma, v, s_{2}\right)\right)-s_{2}\right) \mathrm{e}^{\left.-z_{2} t\left(\sigma, v, s_{2}\right)\right)} \mathrm{d} \beta_{2}(v ; t) .
$$

A strategy-belief profile $(\sigma, \beta)$ constitutes a Perfect Bayesian Equilibrium (PBE) if and only if for all $\left(s_{1}, s_{2}\right) \in\left[s_{1}^{L}, s_{1}^{H}\right] \times\left[s_{2}^{L}, s_{2}^{H}\right]$, and for all $t, u_{i}^{s_{i}}\left(\sigma_{i}, \sigma_{j}, \beta_{i}, t\right) \geq u_{i}^{s_{i}}\left(\sigma_{i}^{\prime}, \sigma_{j}, \beta_{i}, t\right)$

\footnotetext{
${ }^{10}$ If the inefficient offers are made simultaneously then the outcome is defined as a fair lottery over the two offers.
} 
for $i=1,2$ for all right-continuous $\sigma_{i}^{\prime}$, and $\beta_{i}$ is consistent with $\sigma_{j}$, according to Bayes' Rule. ${ }^{11}$

\section{Preliminaries}

In this section, we introduce some preliminary results and notation that will prove to be of great use in the analysis of our mediated bargaining game. We start by showing that equilibrium strategies must be type-monotone: $\mu_{i}^{k}(s) \leq \mu_{i}^{k}\left(s^{\prime}\right)$ for all $s \leq s^{\prime}$, where the inequality is strict unless $\mu_{i}^{k}(s)=0$.

Let $H_{i}^{k}(\sigma, t)$ denote the probability that Player $i$ gives $x^{k}$ no later than $t$ according to $\sigma$. We will write $H_{i}^{k}(t)$ if no confusion arises.

\section{Lemma 1. Any equilibrium strategy must be type-monotone.}

Proof. We prove the lemma in two steps. First, we show weak monotonicity, for which we do not need to assume that strategies are in equilibrium, only that the strategy in question is the best response to some other strategy. Next, we strengthen the result to strict monotonicity when strategies are in equilibrium.

Step 1. The best response to any strategy is such that $\mu_{i}^{k}(s) \leq \mu_{i}^{k}\left(s^{\prime}\right)$ for all $s \leq s^{\prime}$. Fix $\sigma_{2}$ and the associated $H_{2}^{k}(t)$.

Consider Player 1's best response to $\sigma_{2}$. Pick two types of Player 1: $s_{1}<s_{1}^{\prime}$. Denote by $\tau_{1}$ and $\tau_{1}^{\prime}$ the time they concede $x^{k+1}$ and by $t_{k}$ and $t_{k}^{\prime}$ the time they concede $x^{k}$, respectively. We now argue by contradiction, assuming that $t_{k}^{\prime}<t_{k}$.

First, note that the optimal times at which Player 1 will concede the rest of the proposals $x^{l}, l=k-1, \ldots, 1$ do not depend on the fact that whether she conceded $x^{k}$ at time $t=t_{k}$ or $t=t_{k}^{\prime}$. This is so because the optimal time to concede $x^{l}, l=k-1, \ldots, 1$ does not depend on the (observable) history. Hence, it is meaningful to (recursively) define $P_{1}^{k}(s, \tau, t)$ as the expected payoff—evaluated at $\tau \leq t$, where she has already conceded $x^{k+1}$ —of Player 1 of type $s$ conceding $x^{k}$ at time $t$ :

$$
\begin{aligned}
P_{1}^{1}(s, \tau, t)= & \int_{\tau}^{t}\left(x^{2}-s\right) \mathrm{e}^{-z_{1} u} \mathrm{~d} H_{2}^{2}(u)+\left(1-H_{2}^{2}(t)\right)\left(x^{1}-s\right) \mathrm{e}^{-z_{1} t} \\
P_{1}^{k}(s, \tau, t)= & \int_{\tau}^{t}\left(x^{k+1}-s\right) \mathrm{e}^{-z_{1} u} \mathrm{~d} H_{2}^{k+1}(u)+\left(H_{2}^{k}(t)-H_{2}^{k+1}(t)\right)\left(x^{k}-s\right) \mathrm{e}^{-z_{1} t} \\
& +P_{1}^{k-1}\left(s, t, t_{k-1}\right) .
\end{aligned}
$$

\footnotetext{
11 Strictly speaking, this definition is not even a Nash Equilibrium, since we have not described the players' behavior off the equilibrium path. Note, however that given the rules of our game, deviations either end the game or are unobservable. Consequently, we did not find it worthwhile to unnecessarily complicate our definitions solely to satisfy the usual convention.
} 
Because the best response strategy of Player 1 of type $s=s_{1}$ or $s=s_{1}^{\prime}$ maximizes her expected payoff, we immediately have that:

$$
\begin{aligned}
& P_{1}^{k}\left(s_{1}, \tau, t_{k}\right)-P_{1}^{k}\left(s_{1}, \tau, t_{k}^{\prime}\right) \geq 0, \\
& P_{1}^{k}\left(s_{1}^{\prime}, \tau, t_{k}\right)-P_{1}^{k}\left(s_{1}^{\prime}, \tau, t_{k}^{\prime}\right) \leq 0,
\end{aligned}
$$

which implies

$$
P_{1}^{k}\left(s_{1}, \tau, t_{k}\right)-P_{1}^{k}\left(s_{1}, \tau, t_{k}^{\prime}\right) \geq P_{1}^{k}\left(s_{1}^{\prime}, \tau, t_{k}\right)-P_{1}^{k}\left(s_{1}^{\prime}, \tau, t_{k}^{\prime}\right) .
$$

Expanding this inequality, we obtain

$$
\begin{aligned}
& \int_{t_{k}^{\prime}}^{t_{k}}\left(x^{k+1}-s_{1}\right) \mathrm{e}^{-z_{1} u} \mathrm{~d} H_{2}^{k+1}(u)+\Gamma\left(t_{k}, t_{k}^{\prime}\right)\left(x^{k}-s_{1}\right)+\int_{t_{k}}^{t_{k}^{\prime}}\left(x^{k}-s_{1}\right) \mathrm{e}^{-z_{1} u} \mathrm{~d} H_{2}^{k}(u) \\
& \geq \int_{t_{k}^{\prime}}^{t_{k}}\left(x^{k+1}-s_{1}^{\prime}\right) \mathrm{e}^{-z_{1} u} \mathrm{~d} H_{2}^{k+1}(u)+\Gamma\left(t_{k}, t_{k}^{\prime}\right)\left(x^{k}-s_{1}^{\prime}\right) \\
& \quad+\int_{t_{k}}^{t_{k}^{\prime}}\left(x^{k}-s_{1}^{\prime}\right) \mathrm{e}^{-z_{1} u} \mathrm{~d} H_{2}^{k}(u),
\end{aligned}
$$

where $\Gamma\left(t_{k}, t_{k}^{\prime}\right)=\left(H_{2}^{k}\left(t_{k}\right)-H_{2}^{k+1}\left(t_{k}\right)\right) \mathrm{e}^{-t_{k}}-\left(H_{2}^{k}\left(t_{k}^{\prime}\right)-H_{2}^{k+1}\left(t_{k}^{\prime}\right)\right) \mathrm{e}^{-t_{k}^{\prime}}$. Manipulating inequality (4) and dividing by $s_{1}^{\prime}-s_{1}>0$ we have

$$
\int_{t_{k}^{\prime}}^{t_{k}} \mathrm{e}^{-z_{1} u} \mathrm{~d} H_{2}^{k+1}(u) \geq \int_{t_{k}^{\prime}}^{t_{k}} \mathrm{e}^{-z_{1} u} \mathrm{~d} H_{2}^{k}(u)-\Gamma\left(t_{k}, t_{k}^{\prime}\right)
$$

On the other hand, note that by inequality (2)

$$
\int_{t_{k}^{\prime}}^{t_{k}}\left(x^{k+1}-s_{1}^{\prime}\right) \mathrm{e}^{-z_{1} u} \mathrm{~d} H_{2}^{k+1}(u)-\int_{t_{k}^{\prime}}^{t_{k}}\left(x^{k}-s_{1}^{\prime}\right) \mathrm{e}^{-z_{1} u} \mathrm{~d} H_{2}^{k}(u) \leq-\Gamma\left(t_{k}, t_{k}^{\prime}\right)\left(x^{k}-s_{1}^{\prime}\right),
$$

and consequently (we substitute $x^{k+1}$ by $x^{k}$ in the first integral and the inequality remains and becomes strict because we are assuming $t_{k} \geq t_{k}^{\prime}$ )

$$
\int_{t_{k}^{\prime}}^{t_{k}} \mathrm{e}^{-z_{1} u} \mathrm{~d} H_{2}^{k+1}(u)-\int_{t_{k}^{\prime}}^{t_{k}} \mathrm{e}^{-z_{1} u} \mathrm{~d} H_{2}^{k}(u)<-\Gamma\left(t_{k}, t_{k}^{\prime}\right),
$$

a contradiction with (5). We have thus shown weak-type monotonicity.

Step 2. To complete the proof, we now show that in any PBE, there is not a positive mass of types making a concession to $x^{k}$ at any time $t>0$.

Assume otherwise. Then, there is an interval of types of Player 1 making a concession to $x^{k}, k \in\{1, \ldots R-1\}$ at some time $t>0$. Then there must exist a $\delta>0$ such that in the interval $(t-\delta, t)$ no types of Player 2 concede beyond $x^{k+1}$, since by waiting arbitrarily little they can gain a strictly positive amount. However, in equilibrium full concessions must 
be made with positive probability in any time interval as shown by Lemma 3, stated and proved in Appendix A.

Lemma 1 ensures that in equilibrium it is meaningful to talk about the inverse of $\mu(s)$, the time vector of type $s^{\prime}$ s concessions. Let $\varphi_{i}^{k}:[0, \infty) \rightarrow\left[s_{i}^{L}, s_{i}^{H}\right], i=1,2$, such that $\mu_{i}^{k}\left(s_{i}\right)=t>0$ if and only if $\varphi_{i}^{k}(t)=s_{i}$ and $\mu_{i}^{k}\left(s_{i}\right)=0$ if and only if $s_{i} \leq \lim _{t \rightarrow 0^{+}} \varphi_{i}^{k}(t) \equiv$ $\varphi_{i}^{k}(0)$. That is, $\varphi_{i}^{k}(t)$ is the types of Player $i$ who are conceding $x^{k}$ at time $t>0$, while $\varphi_{i}^{k}(0)$ is the supremum of types of Player $i$ who are conceding $x^{k}$ at time $t=0$. At the same time, we can also write $H_{i}^{k}(t)$ as $F_{i}\left(\varphi_{i}^{k}(t)\right)$.

Denote by $\mathcal{Y}$ the set of all the subsets of the feasible agreements, $X$, which include the extreme ones, $x^{1}$ and $x^{R}$. An element $Y$ of $\mathcal{Y}$ is then given by $\left\{y^{1}=x^{1}, y^{2}, \ldots, y^{S}=x^{R}\right\}$, where $2 \leq S \leq R$. We say that a strategy profile is ex post efficient with respect to $Y \in \mathcal{Y}$, if all the (pairs of) types who can trade using prices only in $Y$ eventually do so according to the profile. We say that a strategy "uses" an agreement, if it holds it for a positive length of time.

Lemma 2. Let $Y \in \mathcal{Y}$. The best response to any strategy that exclusively uses feasible agreements in $Y$ is a strategy that exclusively uses feasible agreements in $Y$.

Proof. If the opponent plays as if only $y^{i} \in Y$ were possible, any strategy in which a player proposes $x^{k} \notin Y$ during some positive time interval is strictly dominated by a strategy in which he does not make an intermediate concession. To see this, just observe that maintaining the compromise $x^{k}$ for a while is costly, since upon a concession of the opponent (to some $y^{i} \in Y$ that ends the game), some surplus is lost, while otherwise it makes no difference.

Lemma 2 is instrumental in providing a full characterization of the set of equilibria. In particular, it implies that our game always has a Perfect Bayesian Equilibrium.

Corollary 1. The unique Bayesian equilibrium of the war of attrition is a Perfect Bayesian Equilibrium of the mediated game.

Proof. The existence and uniqueness of equilibrium for $X=\left\{x^{1}, x^{R}\right\}$ is shown in Ponsatí and Sákovics (1995). By Lemma 2, this continues to be an equilibrium for any $Y \in \mathcal{Y}$.

This equilibrium has the undesirable property that it is extremely ex post inefficient: if both types are such that they cannot afford to make a full concession, they never get to agreement even if there exists a feasible price at which they would both gain from trade. Does this mean that mediated bargaining leaves unrealized gains from trade when more than two agreements are possible? Not necessarily. As we show in the next section-while in some cases, the above one is the only equilibrium - an equilibrium where an agreement is reached between any two types who are willing to trade at a feasible price also exists for most "reasonable" parameter values. 


\section{Mediated equilibria}

Let us turn now to the analysis of equilibria where, unlike in the war-of-attrition equilibrium, the possibility to make unobservable concessions does play a role. In these, deals are struck at compromise agreements with positive probability. Henceforth, we restrict attention to strategies that are differentiable almost everywhere.

\subsection{Characterization}

We wish to characterize those equilibria where all types agree on which agreements to skip as they are making concessions. That is, every agreement in some $Y \in \mathcal{Y}$ is either used (held for a positive amount of time) or never conceded, by every type. ${ }^{12}$

Proposition 1. For every $Y \in \mathcal{Y}$, all PBE such that each type uses exactly the agreements in $Y$ (conditional on conceding beyond) are characterized by $2 S-2$ strictly increasing functions $\varphi_{i}^{k}:(0, \infty) \rightarrow\left[s_{i}^{L}, s_{i}^{H}\right], i=1,2$ which must solve the differential system $\left(F_{1}\left(\varphi_{1}^{S}\right)=1\right.$ and $\left.F_{2}\left(\varphi_{2}^{1}\right)=1\right)$ :

$$
\left\{\begin{array}{l}
\dot{\varphi}_{1}^{k}=z_{2} \frac{F_{1}\left(\varphi_{1}^{k+1}\right)-F_{1}\left(\varphi_{1}^{k}\right)}{f_{1}\left(\varphi_{1}^{k}\right)} \frac{1-y^{k+1}-\varphi_{2}^{k+1}}{y^{k+1}-y^{k}}, \quad k=1,2, \ldots S-1, \\
\dot{\varphi}_{2}^{k}=z_{1} \frac{F_{2}\left(\varphi_{2}^{k-1}\right)-F_{2}\left(\varphi_{2}^{k}\right)}{f_{2}\left(\varphi_{2}^{k}\right)} \frac{y^{k-1}-\varphi_{1}^{k-1}}{y^{k}-y^{k-1}}, \quad k=2,3, \ldots, S,
\end{array}\right.
$$

with an initial condition $\left(\varphi_{1}^{1}(0), \ldots \varphi_{1}^{S-1}(0), \varphi_{2}^{2}(0), \ldots, \varphi_{2}^{S}(0)\right)$ satisfying that,

$$
\begin{aligned}
& \text { if } s_{1}^{L}=\varphi_{1}^{1}(0)=\cdots=\varphi_{1}^{l-1}(0)<\varphi_{1}^{l}(0), \text { for some } l=1, \ldots, S-1 \\
& \text { then } \varphi_{2}^{l+1}(0)=\cdots=\varphi_{2}^{S}(0)=s_{2}^{L},
\end{aligned}
$$

and the feasibility condition

$$
\left\{\begin{array}{l}
\varphi_{1}^{1}(t)<\varphi_{1}^{2}(t)<\cdots<\varphi_{1}^{S-1}(t), \\
\varphi_{2}^{S}(t)<\varphi_{2}^{S-1}(t)<\cdots<\varphi_{2}^{2},(t),
\end{array}\right.
$$

for $t>0$.

Proof. Any strategy (relative to $Y \in \mathcal{Y}$ ) of Player 1 of type $s_{1}$ is characterized by a time vector $\left(t_{1}\left(s_{1}\right), t_{2}\left(s_{1}\right), \ldots, t_{S}\left(s_{1}\right)\right)$ that determines the time of concession to every agreement, $y^{k}, k=1, \ldots S$, used in a given equilibrium. ${ }^{13}$

In equilibrium, the time vector for Player 1 of type $s_{1}$, denoted by $\left(t_{1}^{*}, t_{2}^{*}, \ldots, t_{S}^{*}\right)$ (we suppress the dependence on $s_{1}$ for simplicity), must be determined by the following recursive formula (a similar formula applies to Player 2 ): ${ }^{14}$

\footnotetext{
12 The types of potential equilibria that we do not consider are those where over the time the number of agreements used varies.

13 These correspond to the $\mu$ 's of Section 2, dropping the redundant ones.

${ }^{14}$ We denote $F\left(\varphi^{k}(t)\right)$ by $F^{k}(t)$, for simplicity.
} 


$$
\begin{aligned}
t_{1}^{*}=\underset{t \geq t_{2}^{*}}{\operatorname{argmax}}\left\{\int_{t_{2}^{*}}^{t}\left(y^{2}-s_{1}\right) \mathrm{e}^{-z_{1} u} \mathrm{~d} F_{2}^{2}(u)+\left(1-F_{2}^{2}(t)\right)\left(y^{1}-s_{1}\right) \mathrm{e}^{-z_{1} t}\right\}, \\
t_{k}^{*}=\underset{t \in\left[t_{k+1}^{*}, t_{k-1}^{*}\right]}{\operatorname{argmax}}\left\{\int_{t_{k+1}^{*}}^{t}\left(y^{k+1}-s_{1}\right) \mathrm{e}^{-z_{1} u} \mathrm{~d} F_{2}^{k+1}(u)\right. \\
\left.+\left(F_{2}^{k}(t)-F_{2}^{k+1}(t)\right)\left(y^{k}-s_{1}\right) \mathrm{e}^{-z_{1} t}+Q^{k}\left(s_{1}, t\right)\right\}, \quad k>1,
\end{aligned}
$$

where

$$
\begin{aligned}
Q^{2}\left(s_{1}, t\right)= & \int_{t}^{t_{1}^{*}}\left(y^{2}-s_{1}\right) \mathrm{e}^{-z_{1} u} \mathrm{~d} F_{2}^{2}(u)+\left(1-F_{2}^{2}\left(t_{1}^{*}\right)\right)\left(y^{1}-s_{1}\right) \mathrm{e}^{-z_{1} t_{1}^{*}}, \\
Q^{k}\left(s_{1}, t\right)= & \int_{t}^{t_{k-1}^{*}}\left(y^{k}-s_{1}\right) \mathrm{e}^{-z_{1} u} \mathrm{~d} F_{2}^{k}(u)+\left(F_{2}^{k-1}\left(t_{k-1}^{*}\right)\right. \\
& \left.-F_{2}^{k}\left(t_{k}^{*}\right)\right)\left(y^{k-1}-s_{1}\right) \mathrm{e}^{-z_{1} t_{k-1}^{*}}+Q^{k-1}\left(s_{1}, t_{k-1}^{*}\right), \quad k>2 .
\end{aligned}
$$

To see this, note that $t_{1}^{*}$ must be the optimal time to make the final concession, conditional on having made the penultimate concession at $t_{2}^{*}$. This optimum is the result of a trade-off: the gains from waiting are accounted for by the integral, while the losses from waiting are given by the discounted expected value the (final) concession would yield. For earlier concessions, we must include the equilibrium continuation value in the above formula. This is captured by the corresponding $Q$. Since the maximands of the RHS of (10) are differentiable, the first-order condition (when $k>1$ ) is given by

$$
\begin{aligned}
& \mathrm{d} F_{2}^{k+1}(t)\left(y^{k+1}-y^{k}\right) \mathrm{e}^{-z_{1} t}+\left[\mathrm{d} F_{2}^{k}(t)+z_{1}\left(F_{2}^{k+1}(t)-F_{2}^{k}(t)\right)\right]\left(y^{k}-s_{1}\right) \mathrm{e}^{-z_{1} t} \\
& \quad+\left(\frac{\partial Q^{k}\left(s_{1}, t\right)}{\partial t}\right)=0 .
\end{aligned}
$$

Notice that $Q^{k}\left(s_{1}, t\right)$ depends on $t$ only through the lower limit of the integral and therefore

$$
\frac{\partial Q^{k}\left(s_{1}, t\right)}{\partial t}=-\left(y^{k}-s_{1}\right) \mathrm{e}^{-z_{1} t} \mathrm{~d} F_{2}^{k}(t),
$$

reducing the first-order condition to

$$
\mathrm{d} F_{2}^{k+1}(t)\left(y^{k+1}-y^{k}\right)+z_{1}\left[F_{2}^{k+1}(t)-F_{2}^{k}(t)\right]\left(y^{k}-s_{1}\right)=0 .
$$

When $k=1$, similar computations give

$$
\mathrm{d} F_{2}^{2}(t)\left(y^{2}-y^{1}\right)+z_{1}\left[F_{2}^{2}(t)-1\right]\left(y^{1}-s_{1}\right)=0 .
$$

For any $1<k \leq R$, we can derive an analogous first-order condition for Player 2 :

$$
\mathrm{d} F_{1}^{k-1}(t)\left(y^{k}-y^{k-1}\right)+z_{2}\left[F_{1}^{k-1}(t)-F_{1}^{k}(t)\right]\left(1-y^{k}-s_{2}\right)=0 .
$$

Noting that $\mathrm{d} F_{i}^{k}(t)=f_{i}\left(\varphi_{i}^{k}(t)\right) \dot{\varphi}_{i}^{k}$, we obtain system (7).

Moreover, the solutions of system (7) that determine equilibrium paths must have the initial condition (8) because of Lemma 4 (see Appendix A), they must be increasing in 
time (type-monotonicity), and they must satisfy the feasibility condition given by (9) because of the time-monotonicity and the assumption that no agreement in $Y$ is "skipped" by any type.

In the next subsection, we show the existence and uniqueness (for each $Y \in \mathcal{Y}$ ) of equilibria for certain values of the parameters $\left\{s_{1}^{L}, s_{2}^{L}\right\}$ when the number of agreements is three and discuss the existence and uniqueness in the general case.

\subsection{Existence and uniqueness}

Let us first define $T_{i}^{k}=\min \left\{t\right.$, such that $\left.F_{i}^{k}(t)=F_{i}^{k}(\infty)\right\}$. That is, $T_{i}^{k}$ is the earliest time by which Player $i$ proposes at most $y^{k}$ with probability one, conditional on proposing it ever. In this section, we assume differentiability of the density functions.

Proposition 2. For the symmetric game of three possible agreements- $Y=\left\{y^{1}=x^{1}, y^{2}=\right.$ $\left.1 / 2, y^{3}=1-x^{1}\right\}$ and $F_{1}(\cdot) \equiv F_{2}(\cdot)$-there exists a unique ex post efficient equilibrium, such that each type uses exactly the agreements in $Y$, if and only if $s_{1}^{L}=s_{2}^{L}$ is close enough to $y^{1}$.

For the proof of Proposition 2, see Appendix A.

If the distribution of the types is uniform, we need not impose symmetry.

Proposition 3. For the game of three possible agreements- $Y=\left\{y^{1}, y^{2}, y^{3}\right\}$-and uniform-type distributions, there exists a unique ex post efficient equilibrium, such that each type uses exactly the agreements in $Y$, if and only if the pair $\left\{s_{1}^{L}, s_{2}^{L}\right\}$ is close enough to the pair $\left\{y^{1}, 1-y^{3}\right\}$.

For the proof of Proposition 3, see Appendix A.

The above two propositions guarantee the existence and uniqueness of equilibrium paths for specific games with a unique compromise agreement. For the setup of Proposition 3 an ex post efficient equilibrium path is given by $\left\{\varphi_{1}^{1}, \varphi_{1}^{2}, \varphi_{2}^{2}, \varphi_{2}^{3}\right\}$ solving the four-dimensional differential system (7) satisfying the initial condition (8), the feasibility condition (9) and the ex post efficiency condition

$$
\lim _{t \rightarrow T_{1}^{k}} \varphi_{1}^{k}(t)=y^{k}, k=1,2 \text { and } \lim _{t \rightarrow T_{2}^{k}} \varphi_{2}^{k}(t)=1-y^{k}, \quad k=2,3 .
$$

In the setup of Proposition 2 the symmetry simplifies (7) to a two-dimensional differential system.

Both proofs, included in Appendix A, rely on the qualitative theory of differential equations. We show the existence and uniqueness of a solution of the differential system (7) in the appropriate domain, $D=D_{\left\{s_{1}^{L}, s_{2}^{L}\right\}}$, starting at the suitable initial condition and belonging to the stable manifold of the generalized hyperbolic saddle point $\Phi^{*}=\left(y^{1}, y^{2}, 1-y^{2}, y^{3}\right)$. This asymptotic behavior is needed to satisfy ex post efficiency. The proofs also imply that the equilibrium paths correspond to solutions of the differential systems defined for all positive time. 
Remark 1. When asymmetric (or non-uniformly distributed) games with a unique compromise agreement are considered, the major part of the arguments used in the proofs of Propositions 2 and 3 remain valid.

Unfortunately, in general, it is hard to prove that by pulling back the orbits in the stable manifold of the saddle point we cross the suitable boundary to obtain an initial condition satisfying (8); that is, either $\varphi_{1}^{1}(0)=s_{1}^{L}$ and $\varphi_{2}^{3}(0)=s_{2}^{L}$, or $\varphi_{1}^{1}(0)=\varphi_{1}^{2}(0)=s_{1}^{L}$, or $\varphi_{2}^{3}(0)=\varphi_{2}^{2}(0)=s_{2}^{L}$.

Nevertheless, it is natural to claim that the condition $\left\{s_{1}^{L}, s_{2}^{L}\right\}$ "close enough" to $\left\{y^{1}, 1-y^{3}\right\}$ is the suitable condition for existence. In this case, we have uniqueness and $T_{i}^{k}=\infty$ for all $i$ and $k$.

Remark 2. When more than one compromise agreements are available, the main difficulty in showing existence of a (unique) ex post efficient equilibrium is (again) to show, that, given the pair $\left\{s_{1}^{L}, s_{2}^{L}\right\}$, there is an orbit on the stable manifold of the generalized saddle starting at some point satisfying the initial condition given by (8). We may try to argue as before that, as long as the pair of parameters $\left\{s_{1}^{L}, s_{2}^{L}\right\}$ is close enough to the pair $\left\{y^{1}, 1-y^{S}\right\}$, if we pull back the orbits lying on the piece of the stable manifold in the appropriate domain $D=D_{\left\{s_{1}^{L}, s_{2}^{L}\right\}}$, we reach a point compatible with (8). However, when $S>3$, the initial condition given by (8) demands many constraints to be satisfied, a condition difficult to check in a general.

The difficulties discussed in the previous remark prevent us from attempting the statement of a general existence result. This should come as no surprise to the reader familiar with the extreme complexity of multi-dimensional dynamic systems. As it is usual for these problems, further results require a numerical approach. Precisely, as we will see in the next section, when uniform distribution and symmetry is considered, at least from the numerical point of view, solutions exist and are unique for the suitable (close to $y^{1}$ ) values of the parameter $s_{1}^{L}$.

We have thus described all the possible ex post efficient equilibrium paths. However, a priori it is also possible to have ex post inefficient equilibria. As a first step towards their characterization, note that there can be no ex post inefficient equilibrium, such that the $T^{k}$ are finite. To see this, suppose otherwise. Then there would be a positive measure of types who could have traded—say, at $y^{k}$-and have not. However, this strategy cannot be part of an equilibrium since for these types (of either player), proposing $y^{k}$ at $T^{k}$ is a profitable deviation, since they would get to agreement with positive probability. To see this, note that the fact that $y^{k}$ is used in equilibrium implies that a positive measure of types must offer at least $y^{k}$ at some point, and at $T^{k}$ they still must be doing so, by the very fact that they happened not to trade in the candidate equilibrium.

Hence, we must focus on $T^{k}=\infty$. It is then clear that the strategy corresponding to an ex post inefficient equilibrium would be given by a solution of system (7) tending in forward time to one of the singular points on the boundary of $D$ different from the saddle point, and satisfying the monotonicity and feasibility conditions.

When a single compromise agreement is considered, the only singular point different from $\Phi^{*}=\left(y^{1}, y^{2}, 1-y^{2}, 1-y^{3}\right)$ is given by $q=\left(y^{1}, y^{1}, 1-y^{3}, 1-y^{3}\right)$ which has 
(locally) different phase portraits depending on the parameters and the density functions. In particular, as long as condition $S$ given by ${ }^{15}$

$$
\text { condition } S: 1-\frac{4}{y^{3}-y^{2}} \sqrt{\frac{\left(1-F_{1}\left(y^{1}\right)\right)\left(1-F_{2}\left(1-y^{3}\right)\right)}{f_{1}\left(y^{1}\right) f_{2}\left(1-y^{3}\right)}}<0,
$$

is satisfied, there is not any orbit tending to $q$ that satisfies the monotonicity and feasibility conditions.

Condition $S$ guarantees that $q$ has two complex eigenvalues with negative real part. Hence, any orbit tending in forward time to $q$ will spiral around the singular point losing any kind of monotonicity. In general, distributions that do not satisfy this condition will present a very strong asymmetry where most of the types are weak-types lying in the interval $\left(s_{1}^{L}, y^{1}\right)$. In particular, the sufficient condition $S$ is clearly satisfied by uniform distributions, and consequently, Proposition 3 characterizes all equilibria under uniform distributions.

Next, we present an example for which there are orbits tending to an "inefficient" singular point while compatible with monotonicity and feasibility. To do so, we consider the symmetric game $\left\{y^{1}=\frac{1}{4}, y^{2}=\frac{1}{2}, y^{3}=\frac{3}{4}\right\}$ with the density function

$$
f(s)=\frac{a}{\left(s-\left(\frac{1}{5}\right)\right)^{2}},
$$

defined in the interval $\left[s_{1}^{L}, s_{1}^{H}\right]=[0.23,0.75]$. The parameter $a \approx 0.031$ ensures that $f$ is a probability density function. Notice that most of the types lie in the interval $[0.23,0.25]$. System (7) becomes

$$
\begin{aligned}
& \dot{\varphi}_{1}^{1}=\frac{2\left(5 \varphi_{1}^{1}-1\right)\left(\varphi_{1}^{1}-\varphi_{1}^{2}\right)\left(2 \varphi_{1}^{2}-1\right)}{5 \varphi_{1}^{2}-1}, \\
& \dot{\varphi}_{1}^{2}=0.36\left(4 \varphi_{1}^{1}-1\right)\left(\varphi_{1}^{2}-0.75\right)\left(5 \varphi_{1}^{2}-1\right) .
\end{aligned}
$$

It is an exercise to show that the singular point $\left(\frac{1}{4}, \frac{1}{4}\right)$ is an attracting node with the two eigenvectors lying inside $D_{\{0.23\}}$. Fig. 1 gives the numerical plot of the equilibrium paths.

When more than one compromise is used, a similar approach shows that to control the ex post inefficient equilibria we must study all the singular points on the boundary of $D$. Even though the number of singular points is increasing with the number of compromises, there are still only a finite number of local phase portraits that must be considered in order to state the analog of condition $S$ for the general case.

\section{Numerical analysis}

The equilibrium paths are uniquely determined by the integral curves of "large"-dimensional differential systems that, in general, cannot be computed analytically. Hence, it is natural to consider numerical simulations. To do so, we simplify the model to make the computations easier, and to facilitate the comparison of the results for different numbers of

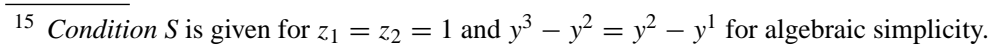




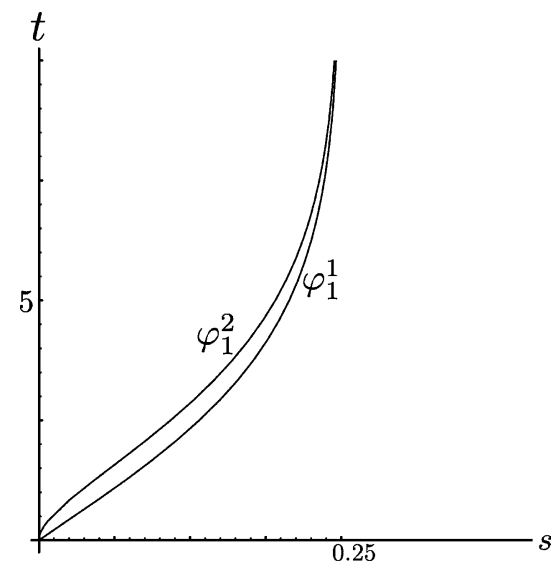

Fig. 1. Numerical plot of an ex post inefficient strategy.

agreements, but still preserving the essence of the game. More precisely, we assume a symmetric game with uniform-type distributions and uniformly distributed feasible agreements (as if we had a smallest money unit). We fix the extreme agreements at $y^{1}=x^{1}=0.25$ and $y^{S}=x^{9}=0.75$ (the value of $S$ will depend on the number of intermediate compromises). We consider the war of attrition, three agreements (with $y^{2}=0.5$ ), five agreements (with $y^{2}=0.375, y^{3}=0.5, y^{4}=0.625$ ), and nine agreements (with $y^{2}=0.3125, y^{3}=0.375$, $\left.y^{4}=0.4375, y^{5}=0.5, y^{6}=0.5625, y^{7}=0.625, y^{8}=0.6875\right)$. Note that as we add more feasible agreements we do not vary the existing ones, to make the comparisons more meaningful. In addition, we assume a common discount rate and normalize it to unity $\left(z_{1}=z_{2}=1\right)$.

The previous section is dedicated to prove general analytic results on the existence (and uniqueness) of the equilibrium paths. The analytic results conclude that the existence of the equilibria depends on the support of the distribution of types. Restricting the distributions to be uniform and symmetric we know that the existence of the equilibrium path depends on the relative distance of the lowest type, $s_{1}^{L}$, and the lowest agreement, $y^{1}=0.25$ (the symmetry forces $s_{1}^{L}=s_{2}^{L}$ ).

We start by considering the WAE. By the assumed symmetry of the game, the equilibrium strategies are symmetric and thus are fully described by a function $\varphi_{1}^{1}(t)$ (for Player 2, we have $\varphi_{2}^{1}(t) \equiv \varphi_{1}^{1}(t)$ ), indicating for each time $t$, the types making total concessions. This function must satisfy the differential equation (see Proposition 1 and Lemma 5):

$$
\dot{\varphi}_{1}^{1}=2\left(0.75-\varphi_{1}\right)\left(0.25-\varphi_{1}^{1}\right),
$$

with the initial condition $\varphi_{1}^{1}(0)=s_{1}^{L}$ and satisfying $\varphi_{1}^{1}(t) \rightarrow \frac{1}{4}$ as $t \rightarrow \infty$. It is now an exercise to check that the unique solution of (14) satisfying the above conditions is given by

$$
\varphi_{1}^{1}(t)=\frac{c \mathrm{e}^{t}-3}{4\left(c \mathrm{e}^{t}-1\right)}
$$


where

$$
c=\frac{0.75-s_{1}^{L}}{0.25-s_{1}^{L}} .
$$

For the equilibria with compromise agreements, because of the symmetry, the $2(S-1)$ dimensional differential system given by (7) simplifies to an $(S-1)$-dimensional differential system. Hence, when $S=3$ ( $S=5$ and 9, respectively), system (7) simplifies to a two ( $S=4$ and 8, respectively)-dimensional differential system. Moreover, each differential system is invariant when considering different values of $s_{1}^{L} \cdot{ }^{16}$

Let us first consider the case of the three symmetric agreements (3-Med) game. System (7) simplifies to the planar system

$$
\begin{aligned}
& \dot{\varphi}_{1}^{1}=4\left(\varphi_{1}^{2}-\varphi_{1}^{1}\right)\left(0.5-\varphi_{1}^{2}\right), \\
& \dot{\varphi}_{1}^{2}=4\left(0.75-\varphi_{1}^{2}\right)\left(0.25-\varphi_{1}^{1}\right) .
\end{aligned}
$$

Moreover, the equilibrium strategies are fully described by the solution(s) of system (15) satisfying the initial condition $\varphi_{1}^{1}(0)=s_{1}^{L}$, the feasibility condition and the terminal condition (derived from ex post efficiency), namely

$$
\lim _{t \rightarrow \infty} \varphi_{1}^{1}(t)=0.25 \text { and } \lim _{t \rightarrow \infty} \varphi_{1}^{2}(t)=0.5 .
$$

A qualitative study of the above differential system shows the existence of three singular points $(0.75,0.75),(0.25,0.25)$ and $(0.25,0.5)$. More precisely, $(0.75,0.75)$ is a repelling node, $(0.25,0.25)$ is an attracting focus, while $(0.25,0.5)$ is a hyperbolic saddle point. Based on the proof of Proposition 2, it is easy to check that the orbit that characterizes the equilibrium path is the branch of the stable manifold leading into the saddle point $(0.25,0.5)$ tangent to the vector $(1,1)$ and lying below the saddle point, coordinate by coordinate. We can find the explicit expression of the stable manifold curve$\varphi_{1}^{1}\left(0.75-\varphi_{1}^{1}\right)+\varphi_{1}^{2}\left(\varphi_{1}^{1}-\varphi_{1}^{2}\right)=0$-because it is an algebraic curve. From here, it is immediate that the existence of the 3-Med equilibrium is restricted to the values of $s_{1}^{L}$ lying in the set $[0,0.25)$, since to the left of that point the integral curve would not have all of its components increasing. In other words, if $s_{1}^{L}<0$, there is no solution of system (15) compatible with condition (16).

For a given $s_{1}^{L} \in[0,0.25)$, we numerically approximate the piece of the stable manifold satisfying $\varphi(0)=s_{1}^{L}$ (not only the curve but its parameterization by $t$, that is the trajectory). To do so, we have used a 7-8 Runge-Kutta method (c.f. Bulirsch and Stoer, 1993). In Fig. 2(a)-(c), the 3-trajectories correspond to the numerical approximations of the equilibrium paths for $s_{1}^{L}=0,0.125$ and 0.1875 , respectively.

\footnotetext{
16 This observation has to be read carefully. The differential system does change because the domain of definition of the differential equation does. However, in the intersection domain, where they are all defined, they coincide.
} 


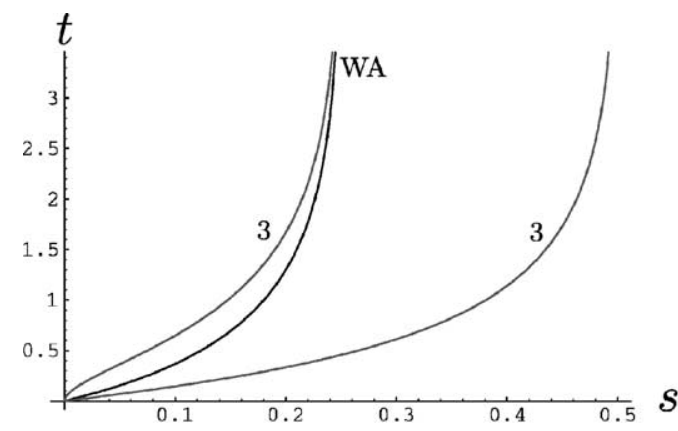

(a)

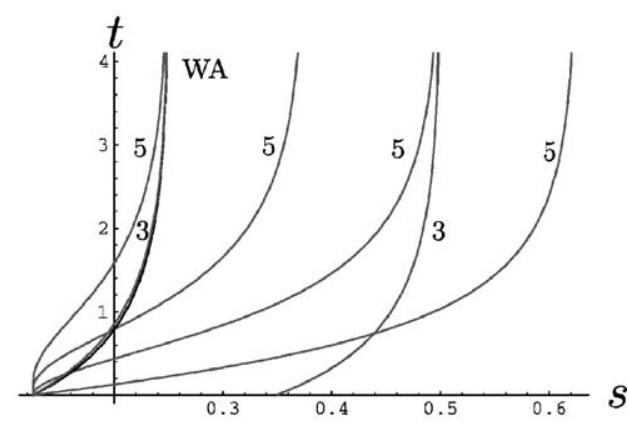

(b)

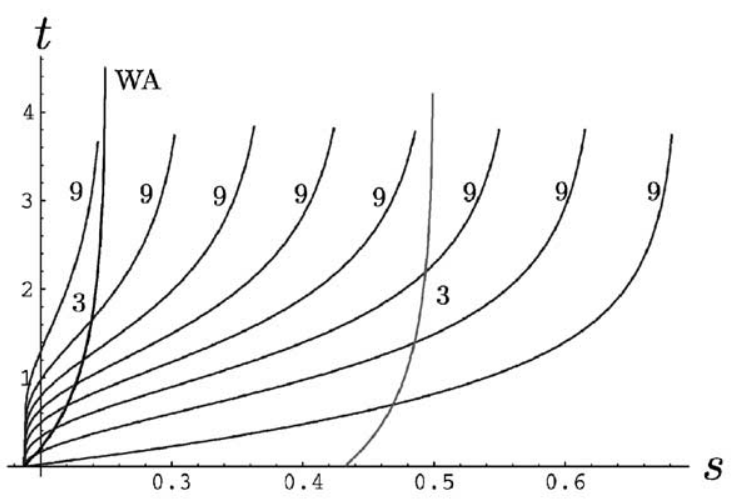

(c)

Fig. 2. The numerical approximation of the equilibrium paths (WA corresponds to the WA equilibrium and the $i$-curves corresponds to the $i$-Med equilibrium, $i=3,5$, 9) when different number of agreements and different values of the parameter $s_{1}^{L}$ are considered. Notice that the full concession of the 3-Med game seems to converge to the WA concession. (a) We draw the WA and 3-Med equilibrium paths when $s_{1}^{L}=0$. (b) We draw the WA, 3-Med and 5-Med equilibrium paths when $s_{1}^{L}=0.125$. (c) We draw the WA, 3-Med and 9-Med equilibrium paths when $s_{1}^{L}=0.1875$. 
Next, we consider the case of the 5 symmetric agreements game (5-Med game). System (7) becomes

$$
\begin{aligned}
& \dot{\varphi}_{1}^{1}=8\left(\varphi_{1}^{2}-\varphi_{1}^{1}\right)\left(0.625-\varphi_{1}^{4}\right), \\
& \dot{\varphi}_{1}^{2}=8\left(\varphi_{1}^{3}-\varphi_{1}^{2}\right)\left(0.5-\varphi_{1}^{3}\right), \\
& \dot{\varphi}_{1}^{3}=8\left(\varphi_{1}^{4}-\varphi_{1}^{3}\right)\left(0.375-\varphi_{1}^{2}\right), \\
& \dot{\varphi}_{1}^{4}=8\left(0.75-\varphi_{1}^{4}\right)\left(0.25-\varphi_{1}^{1}\right),
\end{aligned}
$$

with initial condition ${ }^{17} \varphi_{1}^{1}(0)=\varphi_{1}^{2}(0)=\varphi_{1}^{3}(0)=s_{1}^{L}, s_{1}^{L} \leq \varphi_{1}^{4}(t)<0.625$, and satisfying the feasibility and ex post efficiency conditions.

Similar to the 3-Med case, the above system has a generalized hyperbolic saddle at the point $\Phi=(0.25,0.375,0.5,0.625)$ with a stable manifold of dimension two. However, although the topological structure of the 5-Med game is similar to that of the 3-Med game, the study corresponding to this case is more complicated. In particular, when we considered the 3-Med game, the equilibrium strategy was given by the stable manifold itself, and then it was easy to identify the integral curve (trajectory) satisfying the suitable conditions. Here, the stable manifold is full of trajectories tending to the singular point as $t \rightarrow \infty$ and it is more complicated to identify the one that satisfies a given initial condition.

The numerical experiments and the symmetry of the game show that the 5-Med equilibrium only exists when $s_{1}^{L} \geq 0.125$ (notice that $s_{1}^{L}=0.125$ corresponds to the case where the distance between $s_{1}^{L}$ and $y^{1}$ is equal to the distance between the $y^{i}$ and $y^{i+1}$ ). In Fig. 2(b), the 5-trajectories corresponds to the numerical approximations of the equilibrium paths for $s_{1}^{L}=0.125$.

We finally notice that for the 5-Med game-as well as the 3-Med game-we may easily compute the linear part of the "other" singular points in the boundary of $D$ and show that they involve complex eigenvalues. Thus, the orbits in a neighborhood of those singular points must spiral around and cannot represent equilibrium paths. Therefore, the equilibrium we compute numerically is the only one.

The treatment of the 9-Med equilibrium is similar. It only exists for $s_{1}^{L} \geq 0.1875$. We limit ourselves to displaying the equilibrium 9-strategies in Fig. 2(c).

Using the equilibrium strategies calculated above, we now turn to the calculation and comparison of the ex ante social welfare generated by the different equilibria.

\subsection{Welfare analysis}

The aim of this subsection is to evaluate the relative efficiency of the equilibria differing in the number of agreements used and in the support of the type distribution. A priori, it is not clear what is the specific role of the number of possible agreements relative to the social welfare. On one hand, as this number is larger there are more players getting to agreements - that is, ex post efficiency increases — but, on the other hand, these agreements are realized later.

\footnotetext{
17 They are implied by the symmetry and Lemma 4, stated and proved in Appendix A.
} 
Table 1

Numerical computations of the social welfare depending on the number of possible agreements and the parameter $s_{1}^{L}$

\begin{tabular}{lllll}
\hline$s_{1}^{L}$ & WA & 3-Med & 5-Med & 9-Med \\
\hline 0 & 0.167 & 0.167 & & \\
0.0625 & 0.120 & 0.161 & 0.075 & 0.034 \\
0.125 & 0.075 & 0.128 & 0.063 & 0.047 \\
0.1875 & 0.034 & 0.095 & 0.0625 & \\
0.25 & 0 & 0.063 & \\
\hline
\end{tabular}

The social welfare is represented by the infinite sum (that is, the integral) of the expected payoffs over all types. Then, if in equilibrium two players of types $s_{1}$ and $s_{2}$ agree at time $t\left(s_{1}, s_{2}\right)$ (at any $y \in Y \subset \mathcal{Y} \Rightarrow$, the expected joint payoff associated to this agreement is $W\left(s_{1}, s_{2}\right)=\left(1-s_{1}-s_{2}\right) \mathrm{e}^{-t\left(s_{1}, s_{2}\right)}$. The aggregate expected welfare is then given by

$$
W=\int_{s_{1}^{L}}^{s_{1}^{H}} \int_{s_{2}^{L}}^{s_{2}^{H}} W(x, y) \mathrm{d} F_{2}(y) \mathrm{d} F_{1}(x) .
$$

Since we have assumed a uniform-type distribution, by taking $n$ uniformly distributed types, $s_{i}^{k}, i=1,2 ; k=1, \ldots, n$, in the interval $\left[s_{i}^{L}, s_{i}^{H}\right], i=1,2$, we obtain a natural discrete approximation to this value. The (approximate) social welfare is then given by

$$
\tilde{W}=\frac{1}{n^{2}} \sum_{k=1}^{n} \sum_{l=1}^{n}\left(1-s_{1}^{k}-s_{2}^{l}\right) \mathrm{e}^{-t\left(s_{1}^{k}, s_{2}^{l}\right)} .
$$

Thus, using the equilibrium strategies calculated above, we first obtain numerically $t\left(s_{1}^{k}, s_{2}^{l}\right)$ for each $k$ and $l$ (we have set $n=1000$ ). Next, we use Mathematica to reduce the data and to calculate the sum given in (19). Table 1 gives the expected social welfare approximations for different values of $s_{1}^{L}$ for the four equilibria calculated above: ${ }^{18}$

There are three qualitative features that we can extract from Table 1.

(i) When there exist multiple equilibria, they are Pareto ranked in the following way: The WA is the least efficient and then social welfare is decreasing in the number of compromise agreements used in equilibrium.

(ii) The more agreements are used in equilibrium the smaller is the maximum allowed type support for existence.

(iii) For the lowest $s_{1}^{L}$ such that a given Med equilibrium exists, the WAE and the Med equilibrium induce the same ${ }^{19}$ expected social welfare.

\footnotetext{
18 The above values have been obtained by assuming that we have the same unit mass of types at each interval $\left[s_{1}^{L}, 0.75\right]$. Then the numerical difference between the expected social welfare for different values of $s_{1}^{L}$ is accounted for by the change in the distribution of types (via the support of the density function), rather than by a change in the "number" (or mass) of types.

19 Of course, the values obtained only coincide approximately. However, as we argue below, we believe that the true values indeed are the same.
} 
The fact that the social welfare first increases and then decreases in the number of compromise agreements has a straightforward explanation. As we increase the number of agreements used in equilibrium, two effects arise: first, the ex post efficiency increases, since pairs of types who were unable to get to agreement before, can do so now; second, as it is directly observable from (7), the use of an intermediate agreement slows down the rate of concession to the agreements that were already in use. Now, the first-positive-effect decreases exponentially as we decrease the smallest money unit used, while the second-negative-effect stays constant. Therefore, it is reasonable to have this non-monotonic behavior. ${ }^{20}$

We claim that the underlying game-theoretic reason for the last observation is that to the left of the critical points (in $s_{1}^{L}$ space) it is individually profitable to disregard the possibility of intermediate concessions. Putting it differently, when the opponent is playing the (alleged equilibrium) 3-Med strategy deviating to a strategy that makes only total concessions is profitable. In order to substantiate this claim, we (numerically) show that if $s_{1}^{L}=0$ the expected payoff for Player 2 is the same whether he plays the 3-Med equilibrium strategy or his best strategy ignoring the compromise against the 3-Med equilibrium strategy used by Player 1 .

If Player 2 ignores the compromise, then he maximizes with respect to $t$ the expression

$$
\begin{aligned}
& \int_{0}^{t}\left(1-y^{1}-s\right) \mathrm{e}^{-u} \mathrm{~d} F_{1}^{1}(u)+\left(1-y^{2}-s\right) \mathrm{e}^{-t}\left(F_{1}^{2}(t)-F_{1}^{1}(t)\right) \\
& \quad+\left(1-y^{3}-s\right) \mathrm{e}^{-t}\left(1-F_{1}^{2}(t)\right),
\end{aligned}
$$

that yields the first-order condition

$$
\varphi_{2}(t)=\frac{3 \varphi_{1}^{1}-8 \varphi_{1}^{1} \varphi_{1}^{2}+\left(2 \varphi_{1}^{2}\right)^{2}}{3-4 \varphi_{1}^{1}},
$$

where $\varphi_{1}^{i}$ denotes the strategies of Player 1 in the 3-Med equilibrium ${ }^{21}$ (see Fig. 3). Calculating the expected payoff with the same approximation method as in the previous cases, we obtain that the expected payoff for Player 2 if he ignores the compromise is 0.079 , approximately the same value as the one he would obtain if he played the 3-Med equilibrium strategy (half of the social welfare). ${ }^{22}$

Observation (ii) now follows from observation (i): since the efficiency of the equilibria is decreasing in the number of compromises, the more compromises there are the less profitable the deviation needs to be to upset the equilibrium. But the cost of ignoring a

\footnotetext{
20 The fact that the three-agreement equilibrium is the most efficient one is not a general result: by varying the type distributions the "peak" in efficiency may arrive with more/less agreements.

${ }^{21}$ It is interesting to observe that the deviation strategy involves the full concession of types who, in principle, would obtain a negative payoff doing so $(0.375>0.25)$. This is due to the fact that with some (endogenously determined) probability the opponent is already proposing 0.5 , and thus the total concession will still yield agreement at 0.5 . Consequently, in expected terms, our type can still expect positive utility. The same effect is not present in equilibrium, since there the players concede gradually, so at the time of considering a full concession they are already proposing the compromise agreement and thus they know that the opponent is not offering a compromise at that time.

${ }^{22}$ Again, we have to be careful about the word same. All we can say is that the numerical values differ by less than the precision of the algorithm itself $(0.01)$.
} 


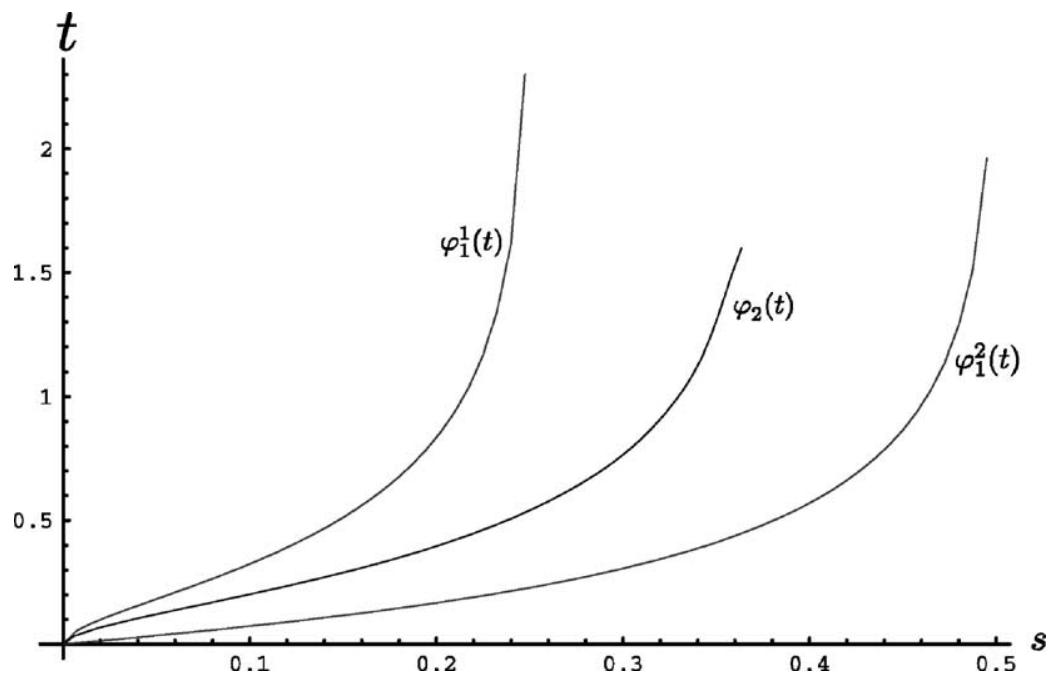

Fig. 3. The numerical approximation of the concession paths when Player 2 plays as if only the two extreme agreements were possible, while Player 1 plays the 3-Med equilibrium strategy.

compromise is directly related to the "weight" of the types who will be impeded from reaching an agreement. Given that the distribution is uniform, increasing this weight is equivalent to increasing the lower bound.

\section{Conclusions}

We have shown that reducing the information flow between negotiators is a powerful tool not only conceptually, but technically as well. We were able to provide a full characterization, and the nature of the multiplicity of our equilibria-it is directly related to the number of agreements used-makes it very easy to resolve: the mediator can simply announce the prices he is willing to accept (which should be the ones that maximize social welfare).

Our results build on the combination of a finite set of equilibrium agreements with a continuous time structure that allows viewing the bargaining process as a game of timing. While the continuous time structure can be relaxed, the assumption that the set of agreements used in equilibrium is finite is technically important and cannot be dispensed with in our proofs. At the same time, the assumption is quite realistic ${ }^{23}$ and, in fact it is reinforced by our results. Recall, that we have shown that the more agreements are used in equilibrium the less efficient the equilibrium is (and the less likely it is to exist). Therefore, the negotiators are naturally driven towards employing "few" prices. ${ }^{24}$ While this reduces ex post efficiency, at no point in time could the mediator induce a Pareto improvement by allowing more prices.

\footnotetext{
23 For a discussion on the appropriateness of a finite set of agreements see van Damme et al. (1990).

24 This concords with the observation that the higher the stakes of a negotiation the higher are the units used.
} 
At the limit, when the set of potential agreements is an interval, things are different. While the equilibria that we have characterized are still supported as such, equilibria where a continuous set of agreements are attained with positive probability may exist as well. Addressing the problem from a point of view complementary to ours, Copic and Ponsatí (2002) show that such equilibria do indeed exist. Instead of assuming that concessions must be discrete they search for equilibria with differentiable patterns of concessions. Their results stand in sharp contrast to ours too: a unique symmetric equilibrium prevails, it is always ex post efficient and independent of the distribution of types.

\section{Acknowledgements}

We acknowledge financial support from the Spanish Ministry of Science and Technology through projects PB98-870, BFM2002-01344 and SEC2000-0684, and from the Government of Catalonia through the projects SGR98-0062 and 2001SGR-00173. Sákovics was at the Institut d'Anàlisi Econòmica when the first drafts were prepared.

\section{Appendix A}

Lemma 3. Until the game ends, in PBE, there is no interval of time without full concessions by both players.

Proof. Assume to the contrary, that Player 1 does not concede to $y^{1}$ during some time interval $\left(t_{0}, t_{1}\right)$, but he does at $t_{1}$. Let $y^{m}$ denote the lowest agreement that he offers with positive probability during this period. Then, by proposing $y^{m}$ or more in the interval $\left(t_{0}, t_{1}\right)$ (and thereby getting to an instant agreement with positive probability), Player 2 cannot maximize payoff: since payoffs are discounted she could increase her payoff by conceding a bit earlier (but still after $t_{0}$ ), while the other possible agreements will not be affected by this deviation (since the opponent does not observe it). Consequently, there will be no agreement at all in $\left(t_{0}, t_{1}\right)$. In that case, however, Player 1 would strictly prefer to make a full concession earlier than $t_{1}$, since there is always a positive measure of types of Player 2 offering $y^{1}$.

The following lemma nails down the initial condition.

Lemma 4. If Player 1 proposes $y^{k}$ with $1 \leq k<S$ (Player 2 proposes $y^{k}$ with $\left.1<k \leq S\right)$ at time $t=0$ with probability $p>0$, respectively, then Player 2 does not propose $y^{l}, l \geq$ $k+1$, (Player 1 does not concede $y^{l}, l \leq k-1$, respectively) at time $t=0$ with positive probability.

Proof. If Player 2 of type $s_{2}$ proposes $y^{l}, l \geq k+1$ at time $t=0$ then his expected payoff is

$$
p\left(1-\frac{1}{2}\left(y^{l}+y^{k}\right)-s_{2}\right)+(1-p) C_{0},
$$

where $C_{0}$ is the continuation value of having proposed $y^{l}$ at time $t=0$. On the other hand, if Player 2 of type $s_{2}$ proposes $y^{l}$ at time $t=\delta>0$ - and so he proposes $y^{k}$ at time $\bar{t} \leq \delta$ - then 
his expected payoff is

$$
p\left(1-y^{k}-s_{2}\right) \mathrm{e}^{-\bar{t}}+(1-p) C_{\delta} \mathrm{e}^{-\delta},
$$

where $C_{\delta}$ is the continuation value of having proposed $y^{l}$ at time $t=\delta$. Now, note that $C_{\delta} \geq C_{0}$, since given that the game is mediated, the opponent cannot condition her strategy on whether the concession has been made and therefore all that can happen in the interval $(0, \delta)$ is that she makes a further concession. The lemma then follows from the fact that if $\delta$ is small enough, $\left(1-y^{k}-s_{2}\right) \mathrm{e}^{-\bar{t}}-\left(1-\frac{1}{2}\left(y^{l}+y^{k}\right)-s_{2}\right)>\epsilon>0$, while $C_{\delta} \mathrm{e}^{-\delta}-C_{0}$ $\geq C_{0}\left(\mathrm{e}^{-\delta}-1\right)$, which tends to zero as $\delta$ tends to 0 .

Lemma 5. If $f_{i}, i=1,2$ are differentiable density functions, the necessary conditions for equilibrium given by (7) characterize maxima.

Proof. In the light of Proposition 1, it is sufficient to prove that the second derivative of the objective function at the critical point (the one satisfying the first-order condition given by (7)) is negative. We only prove here this fact for Player 1 . The proof for Player 2 is similar.

The first derivative of the objective function can be written as (we suppress the dependence on time for simplicity) $\mathrm{e}^{-z_{1} t} A$, where $A$ is the LHS of Eq. (11). Consequently, the second derivative has the form

$$
-z_{1} \mathrm{e}^{-z_{1} t} A+e^{-z_{1} t} \frac{\mathrm{d} A}{\mathrm{~d} t}
$$

Thus, since $A$ is zero at the singular point, we only need to prove that $\mathrm{d} A / \mathrm{d} t$ is negative. Calculating the derivative, we obtain

$$
\begin{aligned}
\frac{\mathrm{d} A}{\mathrm{~d} t}= & {\left[f_{2}^{\prime}\left(\varphi_{2}^{k+1}\right)\left(\dot{\varphi}_{2}^{k+1}\right)^{2}+f_{2}\left(\varphi_{2}^{k+1}\right) \ddot{\varphi}_{2}^{k+1}\right]\left(y^{k+1}-y^{k}\right)+z_{1}\left(f_{2}\left(\varphi_{2}^{k+1}\right) \dot{\varphi}_{2}^{k+1}\right.} \\
& \left.-f_{2}\left(\varphi_{2}^{k}\right) \dot{\varphi}_{2}^{k}\right)\left(y^{k}-s_{1}\right)
\end{aligned}
$$

We can derive the expression of $\ddot{\varphi}_{2}^{k+1}$ by differentiating both sides of the corresponding (functional) equation of system (7). Substituting in and using the fact that $s_{1}=\varphi_{1}^{k}$ at the critical point, we obtain that

$$
\frac{\mathrm{d} A}{\mathrm{~d} t}=-\left(F_{2}\left(\varphi_{2}^{k+1}\right)-F_{2}\left(\varphi_{2}^{k}\right)\right) \dot{\varphi}_{1}^{k}(t)<0,
$$

(where the strict inequality follows by type-monotonicity) as it was desired.

Next we prove Propositions 2 and 3.

Proof of Proposition 2. By Proposition 1 any possible equilibrium path must satisfy the differential system given by (7) and these first-order conditions indeed characterize maxima (see Lemma 5 in Appendix A). In other words, since each type chooses to concede $y^{l}, l=$ 1,2 at the date maximizing her expected payoff, each such solution does indeed characterize a PBE. 
By symmetry, the differential system (7) becomes

$$
\begin{aligned}
& \dot{\varphi}_{1}^{1}=z_{2} \frac{F_{1}\left(\varphi_{1}^{2}\right)-F_{1}\left(\varphi_{1}^{1}\right)}{f_{1}\left(\varphi_{1}^{1}\right)} \frac{\frac{1}{2}-\varphi_{1}^{2}}{\frac{1}{2}-y^{1}}, \\
& \dot{\varphi}_{1}^{2}=z_{1} \frac{1-F_{1}\left(\varphi_{1}^{2}\right)}{f_{1}\left(\varphi_{1}^{2}\right)} \frac{x^{1}-\varphi_{1}^{1}}{\frac{1}{2}-y^{1}} .
\end{aligned}
$$

Consequently, all we have left to show is that (A1) has a unique strictly increasing solution, which, if $s_{1}^{L}$ is close enough to $y^{1}$, satisfies the initial condition $\varphi_{1}^{1}(0)=s_{1}^{L}$ as well as the feasibility condition

$$
s_{1}^{L} \leq \varphi_{1}^{1}(t)<\varphi_{1}^{2}(t)
$$

and the ex post efficiency condition

$$
\lim _{t \rightarrow T_{1}^{1}} \varphi_{1}^{1}(t)=y^{1} \quad \text { and } \quad \lim _{t \rightarrow T_{1}^{2}} \varphi_{1}^{2}(t)=\frac{1}{2} .
$$

It is immediate to show that system (A1) has a singular point at $\Phi^{*}=\left(y^{1}, \frac{1}{2}\right)$ and its linear part is given by the matrix

$$
\left(\begin{array}{ll}
0 & A \\
B & 0
\end{array}\right)
$$

where

$$
A=-z_{2} \frac{F_{1}\left(\frac{1}{2}\right)-F_{1}\left(y^{1}\right)}{f_{1}\left(y^{1}\right)\left(\frac{1}{2}-y^{1}\right)} \quad \text { and } \quad B=-z_{1} \frac{1-F_{1}\left(\frac{1}{2}\right)}{f_{1}\left(\frac{1}{2}\right)\left(\frac{1}{2}-y^{1}\right)},
$$

are both negative. Some computations show that the eigenvalues and the corresponding eigenvectors of the above matrix are given by $\lambda^{ \pm}= \pm \sqrt{A B}$ and $v^{ \pm}=(\mp \sqrt{-A} / \sqrt{-B}, 1)$, respectively (see Perko (1991), for details).

Therefore, the singular point $\Phi^{*}$ of the non-linear system is a saddle point, with a one-dimensional stable and a one-dimensional unstable manifold, $E^{\mathrm{s}}$ and $E^{\mathrm{u}}$, respectively, locally tangent to the linear stable and unstable manifolds $E_{\mathcal{L}}^{\mathrm{s}}$ and $E_{\mathcal{L}}^{\mathrm{s}}$ given by

$$
\begin{aligned}
& E_{\mathcal{L}}^{\mathrm{s}}=\left\{\Phi \in \mathbb{R}^{2} \mid \Phi=\Phi^{*}+\alpha v^{-}, \quad \alpha \in \mathbb{R}\right\}, \\
& E_{\mathcal{L}}^{\mathrm{u}}=\left\{\Phi \in \mathbb{R}^{2} \mid \Phi=\Phi^{*}+\alpha v^{+}, \quad \alpha \in \mathbb{R}\right\} .
\end{aligned}
$$

For a given $s_{1}^{L}$, let us now define the set $D_{\left\{s_{1}^{L}\right\}} \in \mathbb{R}^{2}$ as follows: ${ }^{25}$

$$
D_{\left\{s_{1}^{L}\right\}}=\left\{\Phi \in \mathbb{R}^{2} \mid s_{1}^{L}<\varphi_{1}^{1}<y^{1} \text { and } \varphi_{1}^{1}<\varphi_{1}^{2}<y^{2}\right\} .
$$

It is easy to see that the singular point $\Phi^{*}$ lies on the boundary of $D_{\left\{s_{1}^{L}\right\}}$, and further there is a branch of the stable manifold lying in $D_{\left\{s_{1}^{L}\right\}}$-which is in fact the unique orbit tending to

\footnotetext{
25 We remark that, by changing the values of the parameter $s_{1}^{L}$, the domain where system (A1) is meaningful changes as well. In other words, for a given $s_{1}^{L}$, system (A1) has no meaning when $\varphi_{1}^{k}<s_{1}^{L}, k=1,2$.
} 


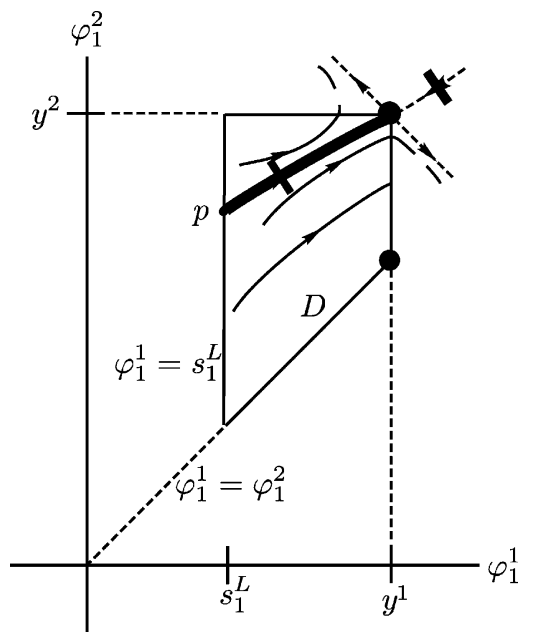

Fig. 4. The phase portrait of system (A1) for a given $s_{1}^{L}$ close to $y^{1}$, near the saddle point and relative to the set $D_{\left\{s_{1}^{L}\right\}}$.

$\Phi^{*}$ in $D_{\left\{s_{1}^{L}\right\}}$-and $\Phi \ll \Phi^{*}$ (where « denotes coordinate by coordinate strict inequality), for all $\Phi \in D_{\left\{s_{1}^{L}\right\}}$ (see Fig. 4). We also note that there are no other singular points in the interior of $D_{\left\{s_{1}^{L}\right\}}$. Finally, the strictly increasing nature of the $F_{i}^{k}$ implies that at every point $\Phi=\left(\varphi_{1}^{1}, \varphi_{1}^{2}\right) \in D_{\left\{s_{1}^{L}\right\}}$ the corresponding vector $\left(\dot{\varphi}_{1}^{1}, \dot{\varphi}_{1}^{2}\right)$ has both components positive. So, if we pull back the orbit passing through any point in $D_{\left\{s_{1}^{L}\right\}}$ we must reach the boundary of $D_{\left\{s_{1}^{L}\right\}}$ given by $\varphi_{1}^{1}=s_{1}^{L}$ or $\varphi_{1}^{1}=\varphi_{1}^{2}$ after a finite time. In particular, this is so if we start at any point in $D_{\left\{s_{1}^{L}\right\}}$ lying on the branch of $E^{S}$.

To end the proof, we claim that for values of the parameter $s_{1}^{L}$ close enough to $y^{1}$, if we pull back the branch of $E^{\mathrm{s}}$ in $D_{\left\{s_{1}^{L}\right\}}$ we cross (first) the boundary of $D_{\left\{s_{1}^{L}\right\}}$ corresponding to $\varphi_{1}^{1}=s_{1}^{L}$. Such a crossing point, (the point $\mathrm{p}$ in Fig. 4) gives the desired initial condition $\left(\varphi_{1}^{1}(0)=s_{1}^{L}, \varphi_{1}^{2}(0) \leq s_{1}^{L}\right)$.

To see the claim, we note that on one hand, the value of $\dot{\varphi}_{1}^{2}$ is uniformly bounded in $D_{\left\{s_{1}^{L}\right\}}$ for all values of the parameter $s_{1}^{L} \in\left(x^{1}-\varepsilon, x^{1}\right)$ (hence, the "vertical" component of the field is bounded), because the density functions are strictly positive on its compact support. On the other hand, considering values of $s_{1}^{L}$ arbitrarily close to $y^{1}$, the compact boundary of $D_{\left\{s_{1}^{L}\right\}}$ given by $\varphi_{1}^{1}=s_{1}^{L}$ is arbitrarily close to $\Phi^{*}$ while the compact boundary of $D_{\left\{s_{1}^{L}\right\}}$ given by $\varphi_{1}^{1}=\varphi_{1}^{2}$ remains far away.

In summary, we have shown that as long as the parameter $s_{1}^{L}$ is close enough to $y^{1}$, there exist a unique orbit starting at $s_{1}^{L}=\varphi_{1}^{1}(0) \leq \varphi_{1}^{2}(0)$, increasing for all $t>0$, and satisfying the feasibility and ex post efficiency condition. ${ }^{26}$ In particular, we have shown that $T_{i}^{k}=\infty, \quad i=1,2, k=1,2$.

\footnotetext{
26 Intuitively, the extreme value of $s_{1}^{L}$ for which the appropriate initial condition holds corresponds to $s_{1}^{L}=$ $\varphi_{1}^{1}(0)=\varphi_{1}^{2}(0)$.
} 
Proof of Proposition 3. From Proposition 1 and Lemma 5, all we have left to show is that (7) has a unique strictly increasing solution, which, if and only if the pair $\left\{s_{1}^{L}, s_{2}^{L}\right\}$ is close enough to the pair $\left\{y^{1}, 1-y^{3}\right\}$, satisfies the initial condition given by (8) as well as the feasibility condition given by (9), and the ex post efficiency condition given by (13).

Assume, first that $\left\{s_{1}^{L}, s_{2}^{L}\right\}=\{0,0\}$ and consequently, the density functions are $f_{1}=$ $1 /\left(s_{1}^{H}\right)$ and $f_{2}=1 /\left(s_{2}^{H}\right)$ on the support $\left[0, s_{1}^{H}\right]$ and $\left[0, s_{2}^{H}\right]$, respectively. In other words, system (7) becomes

$$
\begin{aligned}
& \dot{\varphi}_{1}^{1}=\frac{\left(\varphi_{1}^{2}-\varphi_{1}^{1}\right)\left(1-y^{2}-\varphi_{2}^{2}\right)}{y^{2}-y^{1}}, \\
& \dot{\varphi}_{1}^{2}=\frac{\left(s_{1}^{H}-\varphi_{1}^{2}\right)\left(1-y^{3}-\varphi_{2}^{3}\right)}{y^{3}-y^{2}}, \\
& \dot{\varphi}_{2}^{2}=\frac{\left(s_{2}^{H}-\varphi_{2}^{2}\right)\left(y^{1}-\varphi_{1}^{1}\right)}{y^{2}-y^{1}} \\
& \dot{\varphi}_{2}^{3}=\frac{\left(\varphi_{2}^{2}-\varphi_{2}^{3}\right)\left(y^{2}-\varphi_{1}^{2}\right)}{y^{3}-y^{2}}
\end{aligned}
$$

As a first step, let us rewrite system (7) as

$$
\frac{\mathrm{d} \Phi}{\mathrm{d} t}=H(\Phi)
$$

where $\Phi(t)=\left\{\varphi_{1}^{1}(t), \varphi_{1}^{2}(t), \varphi_{2}^{2}(t), \varphi_{2}^{3}(t)\right\}$. It is immediate to show that system (A6) has a singular point at $\Phi^{*}=\left\{y^{1}, y^{2}, 1-y^{2}, 1-y^{3}\right\}$. The linear part at $\Phi^{*}$ is given by the matrix

$$
D H\left(\Phi^{*}\right)=\left(\begin{array}{cccc}
0 & 0 & -z_{2} & 0 \\
0 & 0 & 0 & -z_{2} \\
-z_{1} & 0 & 0 & 0 \\
0 & -z_{1} & 0 & 0
\end{array}\right),
$$

Some computations show that the eigenvalues and the corresponding eigenvectors are given by $\lambda_{1}^{ \pm}= \pm \sqrt{z_{1} z_{2}}$, both with multiplicity 2 , and $v_{1}^{ \pm}=\left( \pm \sqrt{z_{1} z_{2}} / z_{1}, 0,1,0\right), v_{2}^{ \pm}=$ $\left(0, \pm \sqrt{z_{1} z_{2}} / z_{1}, 0,1\right)$, respectively (see Perko (1991), for details).

Therefore, the singular point $\Phi^{*}$ of the non-linear system, is a generalized saddle with one two-dimensional stable and one two-dimensional unstable manifolds, $E^{\mathrm{s}}$ and $E^{\mathrm{u}}$ locally tangent to the linear stable and unstable manifolds $E_{\mathcal{L}}^{\mathrm{s}}$ and $E_{\mathcal{L}}^{\mathrm{u}}$ given by the equation of the planes

$$
\begin{aligned}
& E_{\mathcal{L}}^{\mathrm{s}}=\left\{\Phi \in \mathbb{R}^{4} \mid \Phi=\Phi^{*}+\alpha_{1} v_{1}^{-}+\alpha_{2} v_{2}^{-} \alpha_{i} \in \mathbb{R}\right\}, \\
& E_{\mathcal{L}}^{u}=\left\{\Phi \in \mathbb{R}^{4} \mid \Phi=\Phi^{*}+\alpha_{1} v_{1}^{+}+\alpha_{2} v_{2}^{+} \alpha_{i} \in \mathbb{R}\right\} .
\end{aligned}
$$

Let us now define the set $D_{\{0,0\}}$ as follows (if no confusions arise we write only $D$ ):

$$
D=\left\{\Phi \in \mathbb{R}^{4} \mid 0<\varphi_{1}^{1}<y^{1} \varphi_{1}^{1}<\varphi_{1}^{2}<y^{2} \varphi_{2}^{3}<\varphi_{2}^{2}<1-y^{2} 0<\varphi_{2}^{3}<1-y^{3}\right\} .
$$


It is easy to see that the singular point $\Phi^{*}$ is on its boundary and $\Phi \ll \Phi^{*}$ (where $\ll$ denotes coordinate by coordinate strict inequality), for all $\Phi \in D$. We also note that there is no other singular point in the interior of $D$.

We claim that $E^{\mathrm{s}} \cap D \neq \emptyset$. To see this, observe that $E^{\mathrm{s}}$ is tangent to $E_{\mathcal{L}^{\mathrm{s}}}^{\mathrm{s}}$ in a neighborhood of $\Phi^{*}$ and, for $\alpha_{1}$ and $\alpha_{2}$ small and negative, the points $\Phi=\Phi^{*}+\alpha_{1} v_{1}^{-}+\alpha_{2} v_{2}^{-} \in\left(E_{\mathcal{L}}^{\mathrm{s}} \cap D\right)$. Next, note that the strictly increasing smooth nature of the $F_{i}^{k}$ implies that every point $\Phi \in$ $D$, is such that the vector $H(\Phi)$ has all its components positive and hence every trajectory in $D$ defines a strictly increasing path at each component (type-monotonicity condition). Moreover, as long as the piece of the stable manifold in $D$ belongs to a small neighborhood of $\Phi^{*}$, it can be represented by the graph of a "flat" function $G=\left(G_{1}, G_{2}\right): \mathbb{R}^{2} \rightarrow \mathbb{R}^{2}$, where $\varphi_{1}^{2}(t)=G_{1}\left(\varphi_{1}^{1}(t), \varphi_{2}^{3}(t)\right)$ and $\varphi_{2}^{2}(t)=G_{2}\left(\varphi_{1}^{1}(t), \varphi_{2}^{3}(t)\right)$. Finally, note that for any point in $E^{\mathrm{s}} \cap D$ the trajectory through that point in forward time ends at $\Phi^{*}$ and it is entirely contained in $D$ (positive time) ${ }^{27}$

Now we chose a pair $\left\{\bar{\varphi}_{1}^{1}, \bar{\varphi}_{2}^{3}\right\}=\left\{\bar{s}_{1}^{L}, \bar{s}_{2}^{L}\right\}$ close enough to the pair $\left\{y^{1}, 1-y^{3}\right\}$ such that the function $G$ gives a unique point $\bar{\Phi} \in E^{s} \cap D$, and let us now consider system (A6) with new uniform distributions $f_{1}$ and $f_{2}$ with supports $\left[\bar{s}_{1}^{L}, s_{1}^{H}\right]$ and $\left[\bar{s}_{2}^{L}, s_{2}^{H}\right]$, respectively. We claim that for such parameter values, $\left\{\bar{s}_{1}^{L}, \bar{s}_{2}^{L}\right\}$ (and corresponding distributions $f_{1}$ and $f_{2}$ ) we have a unique solution of system (A6) satisfying the initial, ex post and feasibility conditions.

To show the claim we notice that in the suitable domain where the two differential systems (the one "defined" by $\left[0, s_{i}^{H}\right]$ and the one defined by $\left[\bar{s}_{i}^{L}, s_{i}^{H}\right], i=1,2$ ) have meaning (notice that, when $\left[\bar{s}_{i}^{L}, s_{i}^{H}\right], i=1,2$ is considered, there is no differential system defined to the "left" of $\bar{s}_{1}^{L}$ and $\bar{s}_{2}^{L}$ ), their integral curves coincide. To see this, we observe that the uniform densities $f_{i}$ are constant functions in their support and they disappear from the equation after simplification (see system (A5)).

To end the proof, we now only need to notice that the orbit starting at the point $\bar{\Phi} \in D$ for the "old system" lies in $D_{\left\{\bar{s}_{1}^{L}, \bar{s}_{2}^{L}\right\}}$ for all $t>0$. So it is an integral curve of the "new" system and satisfies all the increasing, feasibility, initial and ex post efficiency conditions we require. In particular, we have $T_{i}^{j}=\infty$ for all $i$ and $j$.

\section{References}

Abreu, D., Gul, F., 2000. Bargaining and reputation. Econometrica. 68 (1), 85-117.

Admati, A., Perry, M., 1987. Strategic delay in bargaining. Review of Economic Studies 54, 345-364.

Ausubel, L., Deneckere, R., 1992a. Bargaining and the right to remain silent. Econometrica. 60 (3), 597-625.

Ausubel, L., Deneckere, R., 1992b. Durable goods monopoly with incomplete information. Review of Economic Studies 59, 795-812.

Ausubel, L., Deneckere, R., 1993. Efficient sequential bargaining. Review of Economic Studies 60, $435-461$.

Bishop, R.L., 1964. A Zeuthen-Hicks theory of bargaining. Econometrica 32 (3), 410-417.

Broman, E., 1989. The bilateral monopoly model: approaching certainty under the split-the-difference mechanism. Journal of Economic Theory 48, 134-151.

27 To see this, observe that the planes $\left\{\varphi_{1}^{1}=x^{1}, \varphi_{2}^{2}=1-y^{2}\right\}$ and $\left\{\varphi_{1}^{2}=y^{2}, \varphi_{2}^{3}=1-x^{3}\right\}$ are invariant by the flow, contain the eigenvectors associated to the negative real part eigenvalues and the intersection of the stable manifold in a neighborhood of $\Phi^{*}$ with the boundary of $D$ coinciding with these two planes. 
Bulirsch, Stoer, J., 1993. Introduction to Numerical Analysis. Text in Applied Mathematics, vol. 12, second ed. Springer Verlag, Berlin.

Chatterjee, K., Samuelson, L., 1983. Bargaining under incomplete information. Operations Research 31, 835-851.

Chatterjee, K., Samuelson, L., 1987. Bargaining with two-sided incomplete information: an infinite horizon model with alternating offers. Review of Economic Studies 54, 175-192.

Cho, I.-K., 1990. Uncertainty and delay in bargaining. Review of Economic Studies 57, 575-595.

Copic, J., Ponsatí, C., 2002. Filtered bargaining. Institut d'An 1squo;alisi Econ 1squo;omica, mimeo.

Cramton, P., 1984. Bargaining with incomplete information: an infinite-horizon model with two-sided uncertainty. Review of Economic Studies 51, 579-593.

Cramton, P., 1992. Strategic delay in bargaining with two-sided uncertainty. Review of Economic Studies 59, 205-225.

Dunlop, J.T., 1984. Dispute Resolution. Auburn House, Dover, MA.

Fudenberg, D., Tirole, J., 1983. Sequential bargaining with incomplete information. Review of Economic Studies $50,221-247$.

Gresik, T.A., 1996. Incentive-efficient equilibria of two-party sealed-bid bargaining games. Journal of Economic Theory 68, 26-48.

Hicks, J.R., 1932. The Theory of Wages. Macmillan, London.

Inderst, R., 1999. The value of commitment in bargaining. University of Mannheim, mimeo.

Leininger, W., Linhart, P.B., Radner, R., 1989. Equilibria of the sealed-bid mechanism for bargaining with incomplete information. Journal of Economic Theory 48, 63-106.

Perko, L., 1991. Differential Equations and Dynamical Systems. Texts in Applied Mathematics, vol. 7, Springer Verlag, Berlin.

Perry, M., Reny, P.h., 1993. Non-cooperative bargaining with (virtually) no procedure. Journal of Economic Theory 59, 50-77.

Ponsatí, C., 1992. Unique equilibrium in a model of bargaining over many issues. Annales d'Economie et de Statistique 25/26, 81-100.

Ponsatí, C., 1997. Compromise versus capitulation in bargaining with incomplete information. Annales d'Economie et de Statistique 48, 191-210.

Ponsatí, C., Sákovics, J., 1995. The war of attrition with incomplete information. Mathematical Social Sciences 29, 239-254.

Sákovics, J., 1993. Delay in bargaining games with complete information. Journal of Economic Theory 59, 78-95.

Satterthwaite, M., Williams, S., 1989. Bilateral trade with sealed bid k-double auction: existence and efficiency. Journal of Economic Theory 48, 107-133.

van Damme, E., Selten, R., Winter, E., 1990. Alternating bid bargaining with a smallest money unit. Games and Economic Behavior 2, 188-201.

Watson, J., 1998. Alternating-offer bargaining with two-sided incomplete information. Review of Economic Studies 65, 573-594.

Zeuthen, F., 1930. Problems of Monopoly and Economic Warfare. Routledge \& Kegan Paul, London. 